Published in Journal of the Mechanics and Physics of Solids 64 (2014) 411-425

doi: http://dx.doi.org/10.1016/j.jmps.2013.12.008

\title{
Instability of a penetrating blade
}

\author{
D. Bigoni, F. Bosi, F. Dal Corso, and D. Misseroni \\ e-mail: bigoni@ing.unitn.it; federico.bosi@ing.unitn.it; \\ francesco.dalcorso@ing.unitn.it; diego.misseroni@ing.unitn.it \\ ${ }^{a}$ Department of Civil, Environmental and Mechanical Engineering, \\ University of Trento, via Mesiano 77, I-38123 Trento, Italy
}

\begin{abstract}
Application of a dead compressive load at the free end of an elastic rod (the 'blade') induces its penetration into a sliding sleeve ending with a linear elastic spring. Bifurcation and stability analysis of this simple elastic system shows a variety of unexpected behaviours: (i.) an increase of buckling load at decreasing of elastic stiffness; (ii.) a finite number of buckling loads for a system with infinite degrees of freedom (leading to a non-standard Sturm-Liouville problem); (iii.) more than one bifurcation loads associated to each bifurcation mode; (iv.) a restabilization of the straight configuration after the second bifurcation load associated to the first instability mode; (v.) the presence of an Eshelby-like (or configurational) force, deeply influencing stability. Only the first of these behaviours was previously known, the second and third ones disprove common beliefs, the fourth highlights a sort of 'island of instability', and the last one shows surprising phenomena and effects on stability.
\end{abstract}

Keywords: Buckling, Eshelbian forces, material forces, Elastica, smooth contact.

\section{Introduction}

Despite the common belief that structural instability is a fully mature field of mechanics, it has recently been shown that it is still possible to discover new and 'unexpected' phenomena in the critical and postcritical behaviour of simple structures, such as tensile buckling (Zaccaria et al., 2011), buckling inducing shrinking of a structure (Shim et al., 2012), multiple bifurcations in single degree of freedom structures (Bigoni et al., 2012), frictional flutter instability (Bigoni and Noselli, 2011), and that buckling can be exploited to facilitate adhesion (Chan et al., 2008), or to create flexible electronics (Rogers et al., 2010), or to switch a phononic band gap (Bertoldi and Boyce, 2008), or to induce a pattern transformation ( Li et al., 2012).

The aim of the present article is to explore the critical and postcritical behaviour of a simple elastic structure, displaying several unexpected effects, some of which were previously known but received only marginal attention (Feodosyev, 1977; Tarnai, 1980), while others were simply unknown. The importance of the mechanical features highlighted and discovered in the present article lies in the fact that they disprove common believes (for instance, engineers believe that critical loads always increase with the stiffness of a structure, and mathematicians that a bifurcation problem of an elastic rod is always a Sturm-Liouville problem), correct errors in several published works 
(where the so-called 'Eshelby-like force' is wrongly omitted in the calculations), provide a new understanding of the adhesion energy between a structure and a substrate (Majidi, 2007; Majidi et al., 2012), and open the way to unconventional mechanical applications, as for instance to continuous self-restabilizing systems (a simple example of this behaviour, restricted to a system with two degrees of freedom, is reported by Potier-Ferry, 1987). The considered mechanical problem is the following.

A blade (an elastic planar rod) is forced to penetrate into an elastic movable clamp (a frictionless sliding sleeve with a final linear spring) through the application of a dead compressive load at the other edge, Fig. 1.

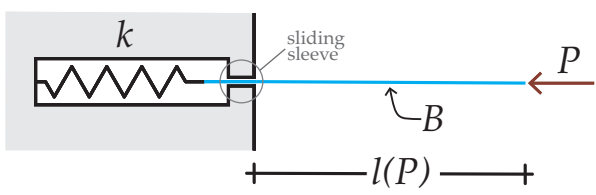

Figure 1: The penetrating blade is an elastic planar rod whose free length $l$ is a function of the amount of the applied axial load $P$. The blade with constant bending stiffness $B$ has a free end subject to the dead load $P$, while at the other edge the blade slides into a frictionless sleeve and is restrained by an axial linear spring of stiffness $k$.

This system is shown to exhibit several surprising and counterintuitive mechanical behaviours, theoretically predicted by elastic analysis and experimentally confirmed through a physical model (Fig. 2, for details of the experimental setup see Sect. 5). These are as follows.
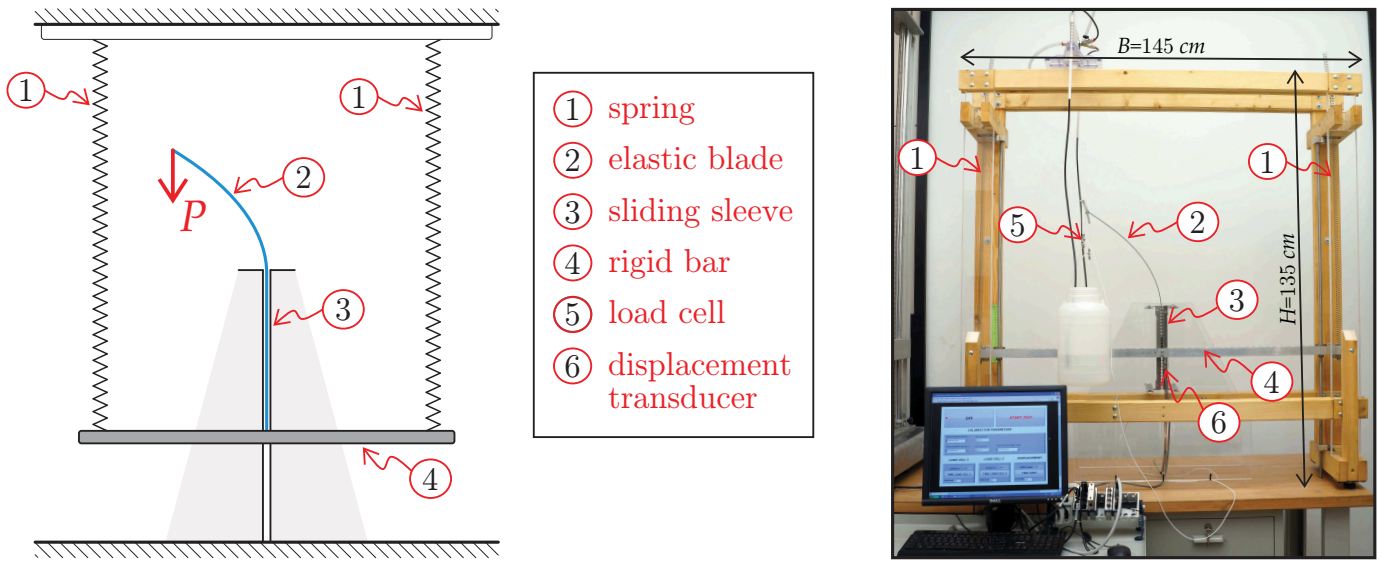

Figure 2: Left: the design scheme employed to realize the structure shown in Fig. 1 and right: its practical realization (prototype 1). Note that the sliding sleeve is borne by a transparent plexiglass support (represented grey on the left). Details of the experimental setup are given in Sect. 5 .

(i.) The buckling load of the system is governed by the relative spring/bending stiffness, so that an increase of elastic stiffness of the spring yields a decrease in the buckling load. Moreover, if the stiffness of the spring is low enough compared to the rod's bending stiffness ('highly compliant' system), there will be no buckling, but only a straight penetration into the sliding 
sleeve. In these conditions an increase in the elastic stiffness of the spring may induce buckling, see Fig. 3 showing that for infinite stiffness (lower part) of the spring the blade buckles, while for highly compliant spring (lower part) the blade does not. Previously, this
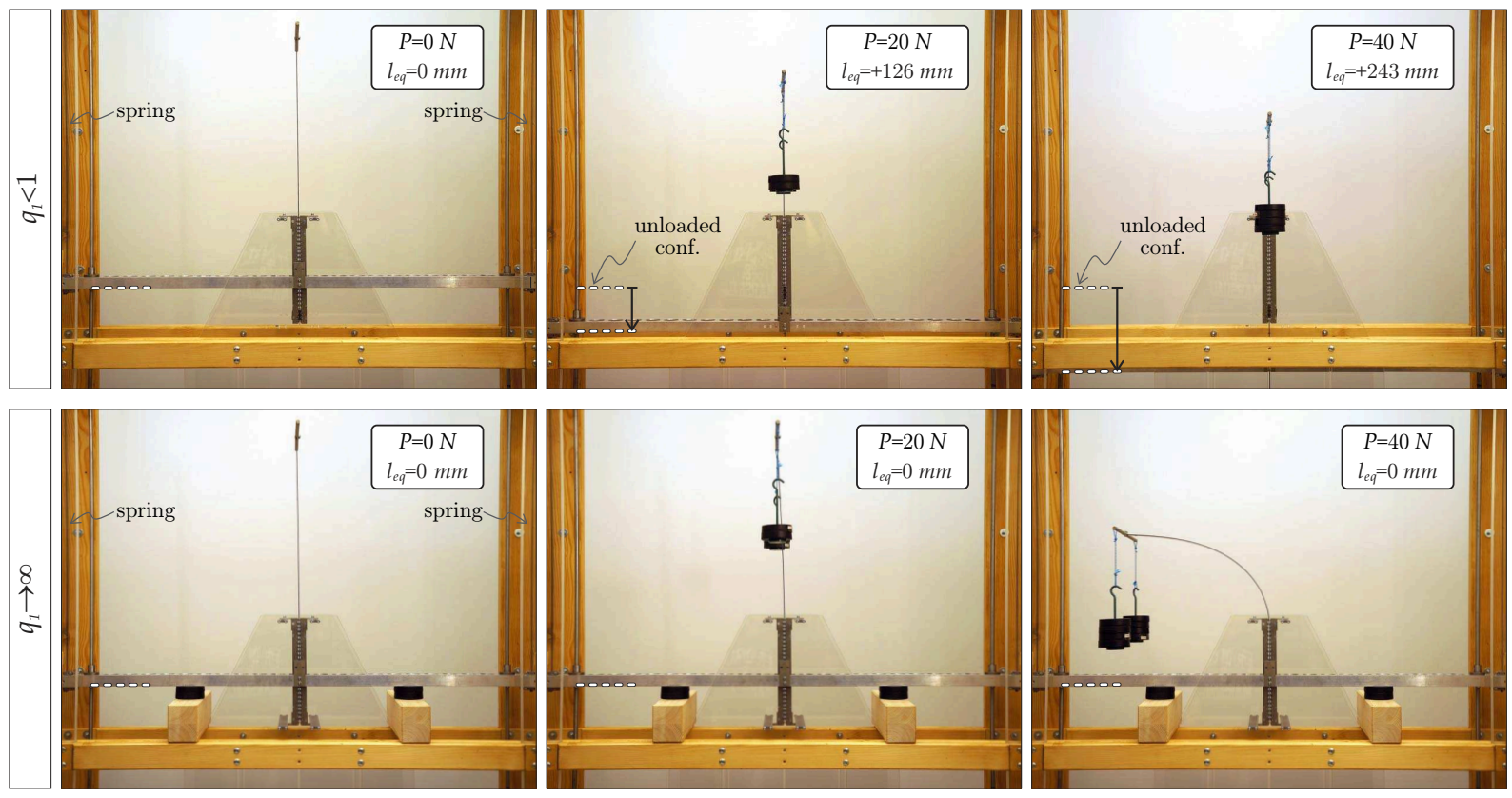

Figure 3: A blade (an elastic planar rod) subject to a dead compressive load $P$ on an edge does not evidence buckling when can penetrate into a sliding sleeve restrained by springs (upper part, so-called 'highly compliant system', $q_{1}<1$ ). The same blade buckles when it is clamped at the lower edge (lower part, $q_{1} \longrightarrow \infty$ ). This model (which can be better understood by analyzing the scheme reported in Fig. 2) shows that an increase of stiffness (the lower clamp is infinitely stiff when compared with the springs restraining the penetration into the sliding sleeve) may lower the buckling load. The length of the blade inside the sliding sleeve at equilibrium is $l_{e q}$, while $q_{1}$ is the dimensionless relative stiffness defined by eq. (2.7).

effect was theoretically noticed by Feodosyev (1977) and on a simpler structure by Tarnai (1980).

(ii.) Buckling of an elastic rod (with ordinary boundary conditions, e.g. doubly pinned) is governed by a Sturm-Liouville problem (Broman, 1970), admitting an infinite number of bifurcation loads. This is commonly associated to the fact that the system 'has infinite degrees of freedom'. Although the system shown in Fig. 1 is continuous, the moving boundary introduced by the sliding sleeve leads to a non-standard Sturm-Liouville problem, so that a finite number of buckling loads is found.

(iii.) Usually, a compressed elastic rod (with ordinary boundary conditions, e.g. doubly pinned) evidences one bifurcation load of the fundamental equilibrium path associated to each bifurcation mode (secondary bifurcations emanating from the postcritical path, as for instance in the case of the doubly-pinned rod, see Bigoni, 2012, Section 1.13.1, are not considered now). The structure shown in Fig. 1 displays two (actually three, but one of these will be shown 
to be physically not accessible) bifurcation loads for the straight configuration associated to each bifurcation mode, a situation occurring also in the simpler system analyzed by Bigoni (2012, Section 1.13.4).

(iv.) In usual structural systems, stability of the fundamental path is lost after buckling and is never 'later' recovered. Differently, the straight configuration of the structure, stable at small load (Fig. 4, upper part on the right), returns to be stable (Fig. 4, lower part on the right) at load sufficiently higher than that corresponding to buckling (more precisely, higher than the load corresponding to a second bifurcation of the fundamental path). This situation occurs also in the simpler systems analyzed by Bigoni (2012, Section 1.13.4) and Potier-Ferry $(1987) \cdot{ }^{1}$
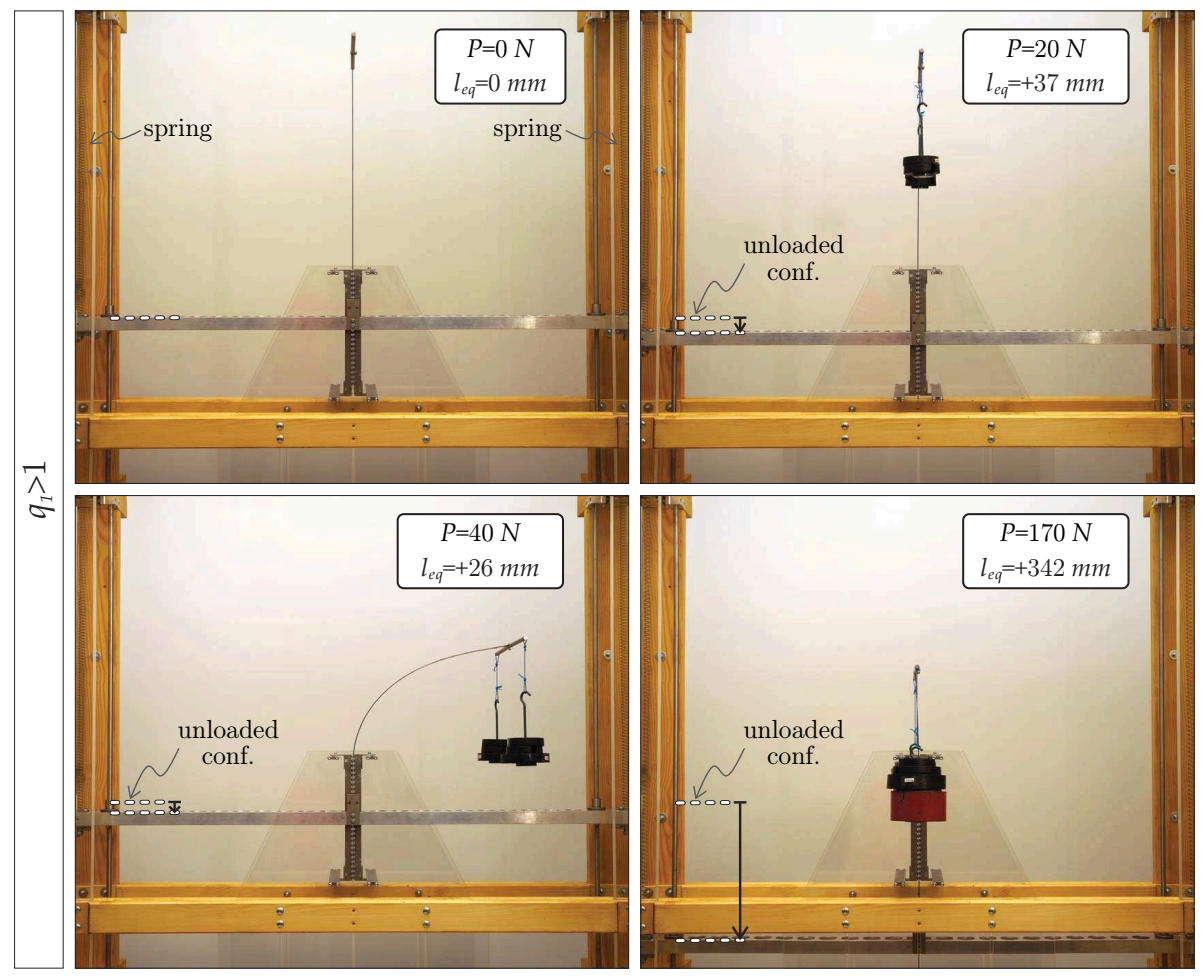

Figure 4: The straight configuration for the blade (elastic planar rod) is stable at small load (upper part, right), becomes unstable at higher load (lower part, left) and, for further increase, it eventually returns stable (lower part, right). The structural model has been realized following the scheme reported in Fig. 2. The length of the blade inside the sliding sleeve at equilibrium is $l_{e q}$, while $q_{1}$ is the dimensionless relative stiffness defined by eq. (2.7).

(v.) Bigoni et al. (2013) have shown that a sliding sleeve constraining the edge of an elastic rod

\footnotetext{
${ }^{1}$ For monotonically increasing loading the structure buckles and does not 'spontaneously' return in the straight configuration. Therefore, the system has to be set in this configuration to observe its stability beyond the second buckling load.

A system that spontaneously self-restabilizes has been shown by Potier-Ferry, 1987.
} 
may induce Eshelby-like (or 'configurational'2) forces in structures, generated by the fact that the rod, freely sliding at one edge, can change its length and therefore release elastic energy. The presence of an Eshelby-like (or configurational) force produced by the sliding sleeve in the structure shown in Fig. 1 strongly affects the post-critical behaviour and its stability. The effects of this force can be counterintuitive, so that the springs in the structure shown in Fig. 5 at low load (upper part on the right, $P=20 N$ ) are subject to an higher elongation than that occurring when a higher load $P$ is applied (lower parts on the left, $P=30 N$, and on the right, $P=40 N$ ). The existence of this Eshelby-like force (erroneously ignored by a
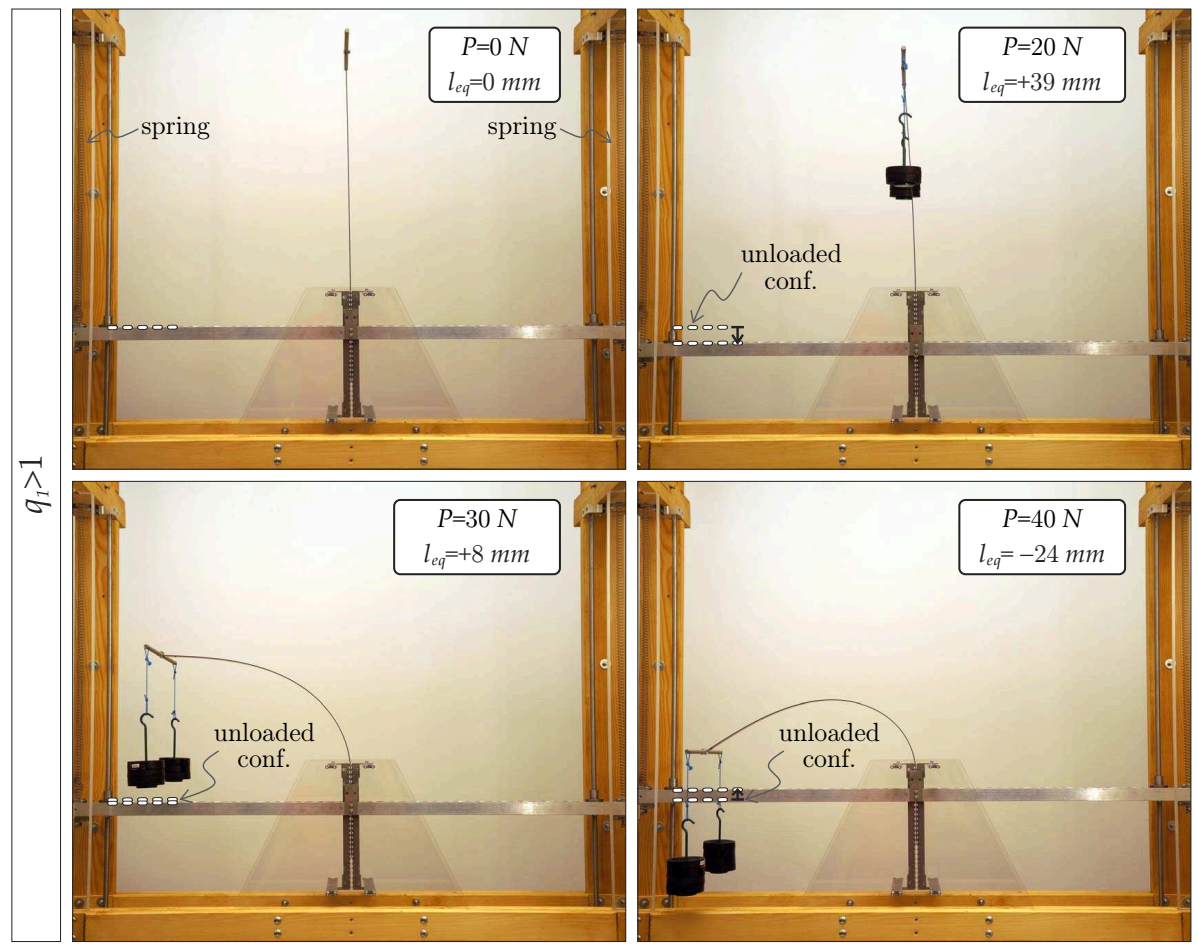

Figure 5: An Eshelby-like force is generated when the blade (elastic planar rod) buckles, so that the length of the rod inserted into the sliding sleeve $\left(l_{e q}\right)$ decreases when the applied compressive load $P$ is increased. As a consequence, the length of the rod inside the sliding sleeve can become even smaller than in the unloaded configuration (i.e. $l_{e q}<0$, lower part, right) with the increasing of the compressive load. This counterintuitive effect is due to the presence of an vertical upward Eshelby-like force generated by the sliding sleeve which is greater than the vertical downward load $P$. The structural model has been realized following the scheme reported in Fig. 2. The length of the blade inside the sliding sleeve at equilibrium is $l_{e q}$, while $q_{1}$ is the dimensionless relative stiffness defined by eq. (2.7).

number of authors in previous articles) becomes even more evident in the photo shown in Fig. 6, because in the absence of this vertical force the equilibrium would be impossible (note that this configuration for the structure is unstable, as will be proven in Sect. 4).

\footnotetext{
${ }^{2}$ These forces have been named by Bigoni et al. (2013) from Eshelby since they can be understood as the derivative of the total potential energy of the system with respect to the configurational parameter characterizing the system, in strict analogy with the concept of Eshelbian force (Eshelby, 1956). 'Configurational force' has here a different meaning than the 'follower' force considered by Bigoni and Noselli (2011).
} 


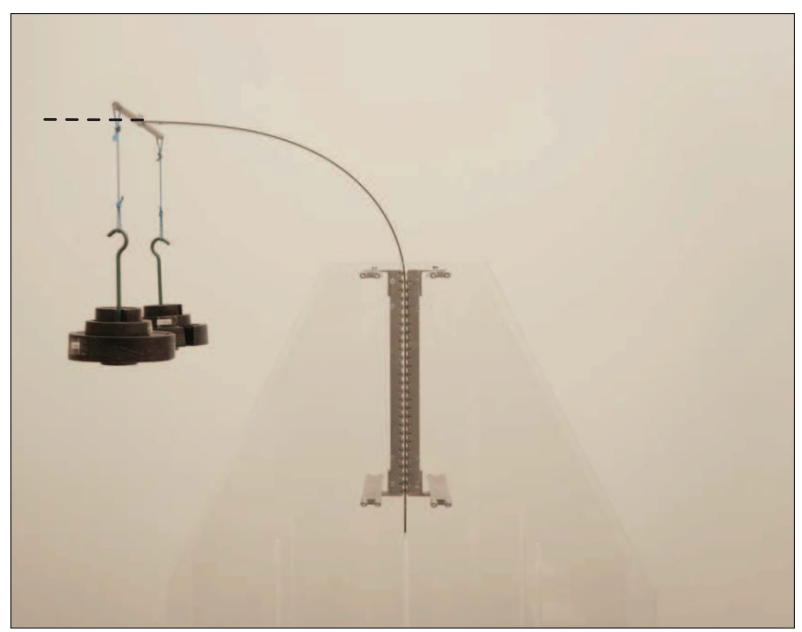

Figure 6: The vertical and upward Eshelby-like force (equal and opposite to the applied downward load) generated by the sliding sleeve makes the equilibrium configuration possible, even if unstable. Note that the tangent to the end of the blade is horizontal, as will be theoretically rationalized in Sect. 3 .

All the above five features are demonstrated in this article, both theoretically and experimentally. In particular, points (i.)-(iii.) are analyzed through a standard bifurcation analysis and integration of the planar elastica. Points (iv.) and (v.) are investigated by using a variational formulation, which allows to confirm the existence of an Eshelby-like force (through consideration of the first variation) and to address stability (through consideration of the second variation).

\section{More than one critical load for each instability mode and finite number of critical loads for continuous elastic systems}

Let us consider an inextensible elastic beam with constitutive behaviour defined by the EulerBernoulli equation

$$
M(s)=B \frac{d \theta(s)}{d s},
$$

where $B$ is the constant bending stiffness, $\theta(s)$ is the angle of inclination of the tangent to the elastica at the curvilinear coordinate $s$. The Euler formula provides the $n$-th critical load (associated to the $n$-th instability mode) for an elastic clamped-free planar rod of length $l$ as

$$
P_{c r, n}=\frac{(2 n-1)^{2} \pi^{2} B}{4 l^{2}}, \quad n \in \mathbb{N}^{+} .
$$

Eq. (2.2) shows that the $n$-th critical load $P_{c r}$ is unique whenever the beam length $l$ is fixed, but this uniqueness may be lost when the length becomes a function of the applied axial load, $l=l(P)$.

If, as shown in Fig. 1, a sliding sleeve with an axial spring replaces the clamp, so that the elastic planar rod (or 'blade') can penetrate into the constraint of an amount $l_{e q}$ (the index 'eq' indicates 
a value at equilibrium $), l(P)$ is given by

$$
l(P)=\bar{l}-l_{e q}(P)
$$

where $\bar{l}$ is the outer length of the blade at null axial load $P, l(P=0)=\bar{l}$. In the particular case of a linear spring with stiffness $k$ and considering the blade in the straight configuration the equilibrium equation in the axial direction is given by

$$
P=k l_{e q},
$$

(an equation that does not hold when the curvature at the point $s=l_{e q}$ is different from zero, $\theta_{e q}^{\prime}\left(l_{e q}\right) \neq 0$, Sect. 3) so that the length of the outer part of the blade is $l(P)=\bar{l}-P / k$ and the Euler formula (2.2) becomes

$$
P_{c r, n}=\frac{(2 n-1)^{2} \pi^{2} B}{4\left(\bar{l}-\frac{P_{c r, n}}{k}\right)^{2}}, \quad n \in \mathbb{N}^{+} .
$$

The solution for the critical load $P_{c r, n}$ from eq. (2.5) leads to the following cubic equation

$$
p_{c r, n}^{3}-2 p_{c r, n}^{2}+p_{c r, n}-\frac{4}{27 q_{n}}=0, \quad n \in \mathbb{N}^{+},
$$

where $p_{c r, n}$ and $q_{n}$ are respectively the dimensionless $n$-th critical load and dimensionless relative stiffness (spring stiffness multiplied by the bar length and divided by a critical load) of the elastic system, the latter given as

$$
q_{n}=\frac{16 k \bar{l}^{3}}{27(2 n-1)^{2} \pi^{2} B}, \quad n \in \mathbb{N}^{+}
$$

and the former is the ' $n$-th' and critical ' $c r$ ' value of the dimensionless load

$$
p=\frac{P}{k l} .
$$

Note that the dimensionless relative stiffness is positive, $q_{n}>0$, defined in a way that for $n=1$ there is no buckling for $q_{1}<1$, and that the dimensionless $n$-th critical load $p_{c r, n}$ has to satisfy the following inequality ${ }^{3}$

$$
p_{c r, n} \leq 1, \quad n \in \mathbb{N}^{+},
$$

corresponding to $l(P) \geq 0$, in other words, to the constraint that the blade cannot buckle after complete penetration into the sliding sleeve.

The solution of the cubic equation (2.6) yields the following conclusions:

\footnotetext{
${ }^{3}$ This restriction holds only for the calculation of the critical loads. Indeed, as it will be shown in Sect. 3 , equilibrium configurations with $p \geq 1$ are possible for non-trivial deformation paths.
} 
i) since all the coefficients of the cubic equation (2.6) are real, the following infinite sequence always exists of real roots

$$
p_{c r, n}^{\mathcal{C}}=\frac{1}{3}\left[2+\sqrt[3]{\frac{q_{n}}{2-q_{n}+2 \sqrt{1-q_{n}}}}+\sqrt[3]{\frac{2-q_{n}+2 \sqrt{1-q_{n}}}{q_{n}}}\right]>1, \quad n \in \mathbb{N}^{+}
$$

all violating the constraint (2.9) and thus representing meaningless solutions from mechanical point of view;

ii) in the case when for a given $m \in \mathbb{N}^{+}$the inequality

$$
q_{m+1}<1<q_{m}, \quad \text { or equivalently } \quad(2 m-1)^{2}<q_{1}<(2 m+1)^{2},
$$

is satisfied, in addition to the sequence of real roots (2.10) other $2 m$ real roots exist for the cubic equation (2.6),

$$
\left.\begin{array}{r}
p_{c r, n}^{\mathcal{A}} \\
p_{c r, n}^{\mathcal{B}}
\end{array}\right\}=\frac{1}{3}\left[2-\frac{1 \pm i \sqrt{3}}{2} \sqrt[3]{\frac{q_{n}}{2-q_{n}+2 \sqrt{1-q_{n}}}}-\frac{1 \mp i \sqrt{3}}{2} \sqrt[3]{\frac{2-q_{n}+2 \sqrt{1-q_{n}}}{q_{n}}}\right],
$$

satisfying the following property

$$
0<p_{c r, n}^{\mathcal{A}} \leq p_{c r, m}^{\mathcal{A}} \leq p_{c r, m}^{\mathcal{B}} \leq p_{c r, n}^{\mathcal{B}}<1, \quad n \leq m \quad n, m \in \mathbb{N}^{+}
$$

so that $2 m$ critical loads are obtained, which correspond to 2 critical loads for the same $n$-th instability mode;

iii) in the particular case when, for a given $m \in \mathbb{N}^{+}, q_{m}=1$ (or, equivalently, $q_{1}=[2 m-1]^{2}$ ), the two real roots associated to the $m$-th mode (2.12) are coincident,

$$
p_{c r, m}^{\mathcal{A}}=p_{c r, m}^{\mathcal{B}}=\frac{1}{3}, \quad m \in \mathbb{N}^{+},
$$

so that $2 m-1$ critical loads are obtained, though $2 m$ postcritical paths still exist.

Dimensionless critical loads, calculated with eq. (2.12), are reported as functions of the dimensionless relative stiffness parameter $q_{1}$ in Fig. 7 for the first three modes $(n=1,2,3)$, together with the experimental data observed on Prototype 1. As it will be shown in the following, the dimensionless load $p_{c r, 1}^{\mathcal{A}}$ corresponds to the lower buckling load and to the loss of stability of the straight configuration, while the dimensionless load $p_{c r, 1}^{\mathcal{B}}$ corresponds to the upper bifurcation load and to the restabilization of the straight configuration. Note that there is no bifurcation for $q_{1}<1$ (Fig. 3 , upper part), but for $q_{1}>1$ there are always two bifurcation loads (the lower called 'buckling' and the higher 'restabilization') associated to the first mode $(n=1)$. A second bifurcation mode emerges at $q_{1}=9$, so that four critical loads exists for $9<q_{1}<25$, two associated with the first mode and two with the second. A third bifurcation mode emerges at $q_{1}=25$. Moreover, while $p_{c r, 1}^{\mathcal{A}}$ corresponds to a critical buckling load for which there is a spontaneous departure from the straight configuration of the blade, $p_{c r, 1}^{\mathcal{B}}$ denotes a load from which the straight configuration returns to be stable, but cannot be spontaneously reached by the blade from its buckled configuration. Finally, we may observe the following. 


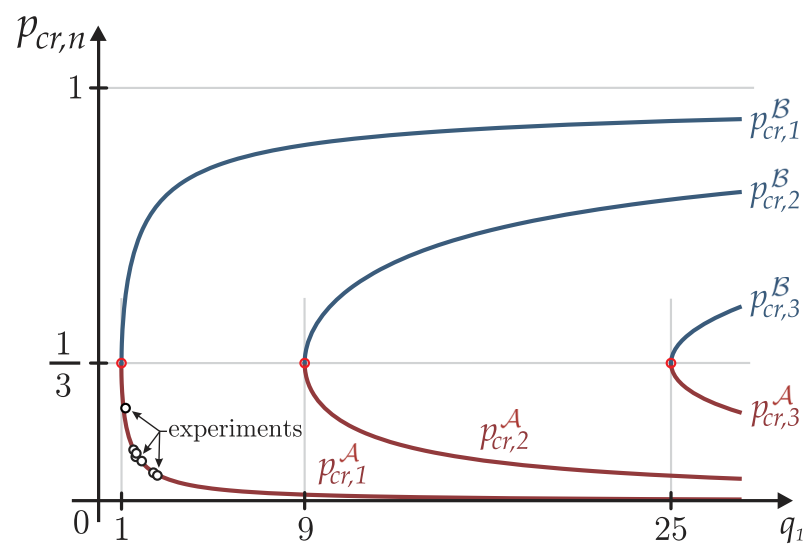

Figure 7: Dimensionless bifurcation loads $p_{c r, n}$ (buckling load $p_{c r, n}^{\mathcal{A}}$ and restabilization load $p_{c r, n}^{\mathcal{B}}$ ) as a function of the dimensionless relative stiffness $q_{1}$ of the elastic system. Note that if the stiffness ratio $q_{1}$ decreases, then the $n$-th buckling load increases while the $n$-th restabilization load decreases, and the number of bifurcation modes can even reduce to zero in the case of 'highly compliant systems' $\left(q_{1}<1\right)$, where bifurcation does not occur. Experimental data (white dots) observed on Prototype 1 are also reported.

i) Considering constant geometrical and material properties of the rod, the reduction of spring stiffness $k$ leads to an increase of the buckling load $p_{c r, 1}^{\mathcal{A}}$ (according to Tarnai, 1980) and a reduction of the restabilization load $p_{c r, 1}^{\mathcal{B}}$;

ii) differently from the usual behaviour of continuous elastic systems, the structure sketched in Fig. 1 has a countable (finite) number (which depends on the elastic properties of the system) of pairs (two for each mode) of critical loads.

\section{From the total potential energy to the equilibrium equations}

An inextensible elastic planar rod (straight in its unloaded configuration, with bending stiffness $B$ and total length $\bar{l}$ ) has one end constrained with a sliding sleeve and a coaxial spring of stiffness $k$, while at the other edge is subject to an axial (dead compressive) force $P$, see Fig. 1. Introducing the curvilinear coordinate $s \in[0, \bar{l}]$ and the rotation field $\theta(s)$ of the planar rod's axis, the axial and transversal displacement fields can be written as

$$
u_{1}(s)=\int \cos \theta(s) d s, \quad u_{2}(s)=\int \sin \theta(s) d s .
$$

With reference to the structural system reported in Fig. 1, the sliding sleeve introduces the condition of null rotation for a portion of the rod,

$$
\theta(s)=0, \quad s \in\left[0, l_{\text {in }}\right],
$$

where $l_{\text {in }}$ is the length of the rod inside the (frictionless, perfectly smooth and bilateral) sliding sleeve, while on the other end of the $\operatorname{rod}(s=\bar{l})$ the boundary condition is

$$
\theta^{\prime}(\bar{l})=0 \text {. }
$$


The total potential energy is

$$
\mathcal{V}\left(\theta, l_{\text {in }}\right)=\int_{l_{\text {in }}}^{\bar{l}} B \frac{\left[\theta^{\prime}(s)\right]^{2}}{2} d s+\frac{1}{2} k\left(l_{\text {in }}\right)^{2}-P\left[\bar{l}-\int_{l_{\text {in }}}^{\bar{l}} \cos \theta(s) d s\right] .
$$

Since the total potential energy (3.4) is written for a system with a moving boundary $l_{i n}$, it is expedient (Courant and Hilbert, 1953, see also Majidi et al. 2012) to introduce a small parameter $\epsilon$ and to take variations (subscript ' $v a r$ ') of an equilibrium configuration (subscript ' $e q$ ') in the form

$$
\theta(s, \epsilon)=\theta_{e q}(s)+\epsilon \theta_{v a r}(s), \quad l_{i n}(\epsilon)=l_{e q}+\epsilon l_{v a r},
$$

with the boundary conditions at the sliding sleeve $\left(s=l_{\text {in }}\right)$

$$
\theta_{e q}\left(l_{e q}\right)=0, \quad \theta\left(l_{e q}+\epsilon l_{\text {var }}\right)=0,
$$

and the boundary conditions at the other end $(s=\bar{l})$

$$
\theta_{e q}^{\prime}(\bar{l})=0, \quad \theta_{\text {var }}^{\prime}(\bar{l})=0 .
$$

A Taylor series expansion of $\theta\left(l_{\text {in }}\right)$ for small $\epsilon$ yields

$$
\theta\left(l_{e q}+\epsilon l_{v a r}, \epsilon\right)=\theta_{e q}\left(l_{e q}\right)+\epsilon\left[\theta_{v a r}\left(l_{e q}\right)+\theta_{e q}^{\prime}\left(l_{e q}\right) l_{v a r}\right]+\frac{\epsilon^{2}}{2} l_{v a r}\left[2 \theta_{v a r}^{\prime}\left(l_{e q}\right)+\theta_{e q}^{\prime \prime}\left(l_{e q}\right) l_{v a r}\right]+\mathcal{O}\left(\epsilon^{3}\right)
$$

so that the boundary conditions (3.6) lead to the following compatibility equations

$$
\theta_{v a r}\left(l_{e q}\right)+\theta_{e q}^{\prime}\left(l_{e q}\right) l_{v a r}=0, \quad 2 \theta_{v a r}^{\prime}\left(l_{e q}\right)+\theta_{e q}^{\prime \prime}\left(l_{e q}\right) l_{v a r}=0 .
$$

Taking into account the Leibniz rule of differentiation and the boundary conditions (3.6) and (3.7), together with compatibility equations (3.9), through integration by parts, the first variation of the functional $\mathcal{V}$ is obtained as

$$
\delta_{\epsilon} \mathcal{V}=-\int_{l_{e q}}^{\bar{l}}\left[B \theta_{e q}^{\prime \prime}+P \sin \theta_{e q}(s)\right] \theta_{v a r}(s) d s+\left[k l_{e q}-P+\frac{B}{2} \theta_{e q}^{\prime}\left(l_{e q}\right)^{2}\right] l_{v a r},
$$

from which, by imposing vanishing for any admissible variation $\theta_{v a r}(s)$ and $l_{\text {var }}$, the elastica is obtained

$$
B \theta_{e q}^{\prime \prime}(s)+P \sin \theta_{e q}(s)=0,
$$

as well as the axial equilibrium condition

$$
P=k l_{e q}+\underbrace{\frac{B}{2}\left[\theta_{\text {eq }}^{\prime}\left(l_{\text {eq }}\right)\right]^{2}}_{\text {Eshelby-likeForce }},
$$

revealing the presence of an Eshelby-like force (Bigoni et al., 2013) generated by the sliding sleeve (see also the asymptotic derivation by Balabukh et al., 1970) and representing the so-called 
'transversality condition' of Courant and Hilbert (1953). Note that, eq. (3.12) reduces to the 'trivial' axial equilibrium equation, eq (2.4), only in the case of null curvature at the sliding sleeve, $\theta_{e q}^{\prime}\left(l_{e q}\right)=0$.

It can be noted from the axial equilibrium equation (3.12) that, the surprising (and never noticed before) equilibrium configuration shown in Fig. 6 is possible in the absence of the spring $(k=0)$, when the elastica is such that the curvature at the sliding sleeve satisfies

$$
P=\frac{B}{2}\left[\theta_{e q}^{\prime}\left(l_{e q}\right)\right]^{2}
$$

corresponding to the fact the dead load $P$ and the Eshelby-like force $\left(B\left[\theta_{e q}^{\prime}\left(l_{e q}\right)\right]^{2} / 2\right)$ are equal and opposite.

\subsection{The elastica}

The rotation field at equilibrium $\theta_{e q}(s)$ is the solution of the following non-linear second-order differential equation with moving boundary condition

$$
\left\{\begin{array}{l}
\frac{d^{2} \theta_{e q}(s)}{d s^{2}}+\lambda^{2} \sin \theta_{e q}(s)=0, \quad s \in\left(l_{e q}, \bar{l}\right) \\
\theta_{e q}\left(l_{e q}\right)=0, \\
\left.\frac{d \theta_{e q}(s)}{d s}\right|_{s=\bar{l}}=0, \\
\lambda^{2}=\frac{k}{B} l_{e q}+\frac{1}{2}\left[\theta_{e q}^{\prime}\left(l_{e q}\right)\right]^{2},
\end{array}\right.
$$

where the normalized axial load $\lambda^{2}=P / B$ has been introduced.

The problem has the trivial solution (denoted with ${ }^{(0)}$ )

$$
\theta_{e q}^{(0)}(s)=0, \quad l_{e q}^{(0)}=\frac{P}{k},
$$

while non-trivial solutions can be obtained through the following procedure. Multiplication of equation $(3.14)_{1}$ by $d \theta_{e q} / d s$ and integration in the variable $s$ yields

$$
\left[\frac{d \theta_{e q}(s)}{d s}\right]^{2}-2 \lambda^{2} \cos \theta_{e q}(s)=\text { constant }
$$

so that, setting $\theta_{e q}(\bar{l})=\theta_{\bar{l}}$ and using the boundary condition (3.14) 3 , it follows (only the solution with the ' + ' sign has been considered)

$$
\frac{d \theta_{e q}(s)}{d s}=\lambda \sqrt{2\left(\cos \theta_{e q}(s)-\cos \theta_{\bar{l}}\right)} .
$$

It is now a standard expedient to operate the following change of variables

$$
\eta=\sin \frac{\theta_{\bar{l}}}{2}, \quad \eta \sin \phi(s)=\sin \frac{\theta_{e q}(s)}{2},
$$


leading to the following differential problem equivalent to the system (3.14)

$$
\left\{\begin{array}{l}
\frac{d \phi(s)}{d s}=\lambda \sqrt{1-\eta^{2} \sin ^{2} \phi(s)}, \\
\phi\left(l_{e q}\right)=h \pi, \\
\phi(\bar{l})=\frac{2 j+1}{2} \pi, \quad h, j \in \mathbb{Z} \\
l_{e q}=\lambda^{2} \frac{B}{k}\left(1-2 \eta^{2}\right) .
\end{array}\right.
$$

Integration of the differential problem (3.19) leads to non-trivial $\eta$, related to the rotation of the loaded end $\theta_{\bar{l}}$ through eq. (3.18), as a function of the load parameter $\lambda$,

$$
(2 n-1) \mathcal{K}(\eta)=\lambda\left[\bar{l}-\lambda^{2} \frac{B}{k}\left(1-2 \eta^{2}\right)\right], \quad n \in \mathbb{N}^{+},
$$

where $n$ corresponds to the number of the instability mode and $\mathcal{K}(\eta)$ is the complete elliptic integral of the first kind,

$$
\mathcal{K}(\eta)=\int_{0}^{\frac{\pi}{2}} \frac{d \phi}{\sqrt{1-\eta^{2} \sin ^{2} \phi}}
$$

Using the dimensionless parameters $p_{n}$ and $q_{n}$, eqs. (2.7) and (2.8), the solution (3.20) can be rewritten in the following form

$$
\left(1-2 \eta^{2}\right)^{2} p_{n}^{3}-2\left(1-2 \eta^{2}\right) p_{n}^{2}+p_{n}-\frac{4}{27 q_{n}}\left[\frac{2 \mathcal{K}(\eta)}{\pi}\right]^{2}=0, \quad n \in \mathbb{N}^{+},
$$

which is a cubic equation providing in general three 'deformation paths' $\left(\mathcal{A}_{n} ; \mathcal{B}_{n} ; \mathcal{C}_{n}\right)$ corresponding to the $n$-th mode, namely, three dimensionless loads $\left(p_{n}^{\mathcal{A}} ; p_{n}^{\mathcal{B}} ; p_{n}^{\mathcal{C}}\right)$ for each mode as functions of the rotation at the loaded end $\theta_{\bar{l}}$, through inversion of relation $(3.18)_{1}$, and the relative dimensionless stiffness $q_{n}$

$$
p_{n}^{\mathcal{A}}=p_{n}^{\mathcal{A}}\left(\theta_{\bar{l}}, q_{n}\right), \quad p_{n}^{\mathcal{B}}=p_{n}^{\mathcal{B}}\left(\theta_{\bar{l}}, q_{n}\right), \quad p_{n}^{\mathcal{C}}=p_{n}^{\mathcal{C}}\left(\theta_{\bar{l}}, q_{n}\right), \quad n \in \mathbb{N}^{+}
$$

Note that in the limit of undeformed configuration (implying a null angle at the loaded end $\theta_{\bar{l}} \rightarrow$ 0 , so that $\eta \rightarrow 0, \mathcal{K}(\eta \rightarrow 0) \rightarrow \pi / 2)$, the cubic equation (3.22) corresponds to that obtained within the small displacement theory, eq. (2.6), and again yields the critical dimensionless loads $\left(p_{c r, n}^{\mathcal{A}} ; p_{c r, n}^{\mathcal{B}} ; p_{c r, n}^{\mathcal{C}}\right)$, given by eqs. $(2.10)$ and $(2.12)$.

For a given value of the applied load, the rotation at the loaded end (related to the three 'deformation paths') can be computed from eq. (3.22) and then, through inversion of relation $(3.18)_{1}$, the corresponding rotation field can be obtained

$$
\theta_{e q}(s)=2 \arcsin \left[\eta \operatorname{sn}\left(\lambda\left(s-l_{e q}\right), \eta\right)\right]
$$


from which the axial and transversal displacements are obtained by integration, eq. (3.1), as

$$
\begin{aligned}
& u_{1}(s)=-s+\frac{2}{\lambda} E\left[\operatorname{am}\left(\lambda\left(s-l_{e q}\right), \eta\right), \eta\right]-l_{e q}, \\
& u_{2}(s)=\frac{2 \eta}{\lambda}\left[1-\operatorname{cn}\left(\lambda\left(s-l_{e q}\right), \eta\right)\right],
\end{aligned}
$$

which can be evaluated at the loaded end, thus yielding

$$
u_{1}(\bar{l})=\frac{2}{\lambda}[E(\eta)-\mathcal{K}(\eta)]-l_{e q}, \quad u_{2}(\bar{l})=\frac{2 \eta}{\lambda} .
$$

In eq. (3.25) the functions am, cn and sn denote the Jacobi amplitude, Jacobi cosine amplitude and Jacobi sine amplitude functions,

$$
\operatorname{cn}(x, \eta)=\cos [\operatorname{am}(x, \eta)], \quad \operatorname{sn}(x, \eta)=\sin [\operatorname{am}(x, \eta)],
$$

while $E(x, \eta)$ is the incomplete elliptic integral of the second kind of modulus $\eta$, defined as

$$
E(x, \eta)=\int_{o}^{x} \sqrt{1-\eta^{2} \sin ^{2} t} d t .
$$

Finally, in the case when the stiffness of the axial spring vanishes $(k=0$, Fig. 6$)$, eq. (3.13) represents the only possible equilibrium configuration of the system. This equation can be rewritten, by introducing a change of variables in eq. (3.18), as

$$
1-2 \sin ^{2}\left(\frac{\theta_{\bar{l}}}{2}\right)=0
$$

Eq. (3.29) reveals that the only equilibrium configuration for the system without spring occurs when the end tangent to the rod is orthogonal to the sliding sleeve, $\theta_{\bar{l}}=\pi / 2$, representing a purely geometrical condition, visibly satisfied in Fig. 6.

\section{$4 \quad$ Stability of configurations}

In order to evaluate the stability of the equilibrium configurations for the penetrating blade, the second variation of the functional $\mathcal{V}$, eq. (3.4), has to be calculated. Considering the boundary conditions (3.6) and the perturbations in the rotation field $\theta_{v a r}(s)$ and in the length $l_{\text {var }}$ satisfying the compatibility equations (3.9), the second variation evaluated at an inflexed equilibrium configuration can be written as

$$
\delta_{\epsilon}^{2} \mathcal{V}=\frac{1}{2}\left\{k l_{\text {var }}^{2}+B \int_{l_{\text {eq }}}^{\bar{l}}\left[\theta_{\text {var }}^{\prime}(s)\right]^{2} d s-P \int_{l_{\text {eq }}}^{\bar{l}}\left[\theta_{\text {var }}(s)\right]^{2} \cos \theta_{e q}(s) d s\right\}
$$


The stability or instability of an equilibrium configuration is then related to the sign of the second variation $\delta_{\epsilon}^{2} \mathcal{V}$, evaluated for that equilibrium configuration, namely,

$$
\delta_{\epsilon}^{2} \mathcal{V}= \begin{cases}>0 & \text { stable equilibrium configuration, } \\ <0 & \text { unstable equilibrium configuration, }\end{cases}
$$

for any admissible perturbations $\theta_{\text {var }}(s)$ and $l_{\text {var }}$ satisfying the compatibility eqs. (3.9).

Considering the auxiliary function $\Gamma(s)$, solution of the following boundary value problem (the Riccati equation plus a boundary condition, see van Brunt, 2005)

$$
\left\{\begin{array}{l}
\frac{\partial \Gamma(s)}{\partial s}-P \cos \theta_{e q}(s)-\frac{\Gamma(s)^{2}}{B}=0 \\
\Gamma(\bar{l})=0
\end{array}\right.
$$

the compatibility eqs. (3.9) and the following identity

$$
\int_{l_{e q}}^{\bar{l}} \frac{d}{d s}\left[\theta_{v a r}^{2}(s) \Gamma(s)\right] d s-\left[\theta_{v a r}^{2}(s) \Gamma(s)\right]_{l_{e q}}^{\bar{l}}=0,
$$

the second variation, eq. (4.1), can be rewritten as

$$
\delta_{\epsilon}^{2} \mathcal{V}=\frac{1}{2}\left\{B \int_{l_{\text {eq }}}^{\bar{l}}\left[\theta_{\text {var }}^{\prime}(s)+\frac{\Gamma(s)}{B} \theta_{\text {var }}(s)\right]^{2} d s+l_{\text {var }}^{2}\left[\left[\theta_{\text {eq }}^{\prime}\left(l_{e q}\right)\right]^{2} \Gamma\left(l_{e q}\right)+k\right]\right\}
$$

To judge the stability of the equilibrium configurations, the two conditions obtained by Majidi et al. (2012) are exploited here. In particular (note that the existence of a bounded $\Gamma(s)$ on the interval $\left[l_{e q}, \vec{l}\right]$ implies that the integral in (4.5) is non-negative)

- Necessary condition (N) for the equilibrium configuration defined by $\left\{\theta_{e q}(s), l_{e q}\right\}$ to be stable (so that it minimizes the functional $\mathcal{V}$ ) is that the auxiliary function $\Gamma(s)$, solution of to the boundary-value problem (4.3), cannot become unbounded in the interval $\left[l_{e q}, \bar{l}\right]$ and satisfies the following inequality

$$
\Delta=\left[\theta_{e q}^{\prime}\left(l_{e q}\right)\right]^{2} \Gamma\left(l_{e q}\right)+k \geq 0 .
$$

- Sufficient condition (S) for the trivial equilibrium configuration $\left\{\theta_{e q}(s)=0, l_{e q}=P / k\right\}$, to be stable is that the auxiliary function $\Gamma(s)$, solution of the boundary-value problem (4.3), is bounded.

In order to obtain the auxiliary function $\Gamma(s)$, it is instrumental to consider the Jacobi transformation

$$
\Gamma(s)=-B \frac{\Lambda^{\prime}(s)}{\Lambda(s)}
$$


leading to the following Jacobi boundary value problem

$$
\left\{\begin{array}{l}
\Lambda^{\prime \prime}(s)+\lambda^{2} \cos \theta_{e q}(s) \Lambda(s)=0 \\
\Lambda(\bar{l})=1 \\
\Lambda^{\prime}(\bar{l})=0
\end{array}\right.
$$

Once the function $\Gamma(s)$ and the auxiliary function $\Lambda(s)$ are obtained for a specific equilibrium configuration by solving the differential eqs. (4.3) and (4.8), the stability of that equilibrium configuration can be judged through the necessary $(\mathrm{N})$ and sufficient $(\mathrm{S})$ conditions.

\subsection{Stability of trivial configurations}

In the case when the equilibrium configuration is straight, identifying the trivial solution $\left\{\theta_{e q}(s)=0\right.$; $\left.l_{e q}=P / k\right\}$, the Jacobi boundary value problem (4.8) simplifies to

$$
\left\{\begin{array}{l}
\Lambda^{\prime \prime}(s)+\lambda^{2} \Lambda(s)=0 \\
\Lambda(\bar{l})=1 \\
\Lambda^{\prime}(\bar{l})=0
\end{array}\right.
$$

which, through the Jacobi transformation (4.7), leads to the following solution for the auxiliary function $\Gamma(s)$

$$
\Gamma(s)=\sqrt{P B} \tan \left(\sqrt{\frac{P}{B}}(\bar{l}-s)\right) .
$$

The auxiliary function $\Gamma(s)$, eq. (4.10), is bounded for $s \in\left[l_{e q}=P / k, \bar{l}\right]$ if and only if

$$
\frac{P}{B}\left(\bar{l}-\frac{P}{k}\right)^{2}<\frac{\pi^{2}}{4}
$$

which can be rewritten in the dimensionless form

$$
p(1-p)^{2}<\frac{4}{27 q_{1}}
$$

an equation reducing to eq. (2.6) when ' $<$ ' is replaced by '='. Considering now the necessary $(\mathrm{N})$ and sufficient (S) conditions, the trivial straight configuration is:

- for $q_{1}<1$ : stable ('highly compliant' systems, see eq. (2.7));

- for $q_{1}>1$ :

$$
\begin{array}{ll}
\text { - stable } & \text { if } P<P_{c r, 1}^{\mathcal{A}}, \\
\text { - unstable } & \text { if } P_{c r, 1}^{\mathcal{A}}<P<P_{c r, 1}^{\mathcal{B}}, \\
\text { - stable } & \text { if } P_{c r, 1}^{\mathcal{B}}<P<k \bar{l} .
\end{array}
$$




\subsection{Stability of non-trivial configurations}

In the case of non-trivial equilibrium configurations, $\theta_{e q}(s) \neq 0$, the function $\Lambda(s)$, solution of the Jacobi problem (4.8), can be obtained only through numerical integration. Numerical investigations performed varying the dimensionless relative stiffness $q_{1}$ and the applied load $P$ show which nontrivial configuration is unstable, through application of the necessary condition $(\mathrm{N})$. In particular, a configuration is unstable either $\left(\mathrm{N}_{1}\right)$ when the function $\Lambda(\hat{s})=0$ with $\hat{s} \in\left[l_{e q}, \bar{l}\right)$, namely, the auxiliary function $\Gamma(s)$, is bounded, or $\left(\mathrm{N}_{2}\right)$ when $\Delta<0$, see eq. (4.6).

It is concluded that unstable equilibrium configurations are:

- all the investigated non-trivial equilibrium configurations of the paths $\mathcal{A}_{n}, \mathcal{B}_{n}$, and $\mathcal{C}_{n}$ with $n \geq 2$, due to condition $\left(\mathrm{N}_{1}\right)$;

- all the non-trivial equilibrium configurations of the path $\mathcal{B}_{1}$, due to condition $\left(\mathrm{N}_{2}\right)$;

- all the non-trivial equilibrium configurations of the path $\mathcal{A}_{1}$, due to condition $\left(\mathrm{N}_{2}\right)$, when $q_{1}<1$

- the non-trivial equilibrium configurations associated to a negative slope in the $\theta_{\bar{l}}-P$ plane (snap-through instability) of the path $\mathcal{A}_{1}$, due to condition $\left(\mathrm{N}_{2}\right)$, and occurring only when $1<q_{1}<q^{S T} \approx 1.6875$.

All other non-trivial equilibrium configurations not listed above satisfy the necessary condition $(\mathrm{N})$ and therefore could be stable, although this cannot be proven and remains an open problem. However, although stability for these cases cannot - for the moment- be rigorously decided, the stability of the non-trivial equilibrium configurations $\mathcal{A}_{1}$ has been experimentally confirmed (see the experimental results presented in the next Section).

Finally, it is straightforward to judge the stability of the system in the case when the axial spring is absent $(k=0$, Fig. 6$)$ and conclude that, although the auxiliary function $\Gamma(s)$ is always bounded, all the possible equilibrium configurations are unstable due to condition $\left(\mathrm{N}_{2}\right)$.

\section{$5 \quad$ Theory vs. experiments}

The buckling loads of the structure sketched in Fig. 1 are given by Eq. (2.12). Moreover, for a given value of the applied load $P, \lambda$ is known from its definition $\left(\lambda^{2}=P / B\right)$, so that Eq. (3.20) allows the calculation of the corresponding $\eta$ and thus $l_{e q}$ is known from Eq. (3.19) $)_{4}$. Finally, Eqs. (3.25) give the components of the elastica and, in particular, Eqs. (3.26) permit the evaluation of the displacement components of the blade's end point. Instability (stability) of the configurations has been determined using condition N (condition S) obtained in Sect. 4. As we have already mentioned in that Section, the stability of the ascending branches of the first-mode of postcritical behaviour (where the necessary condition for stability is verified) has been only conjectured on the basis of our experimental results, while stability and instability of all the rest of the trivial and bifurcated paths has been rigorously proven.

Restricting the attention for the moment only to the first bifurcation mode of the structure shown in Fig. 1, the dimensionless load $p=P /(k \bar{l})$ is plotted as a function of the dimensionless displacement components $\left\{u_{1}(\bar{l}) / \bar{l}, u_{2}(\bar{l}) / \bar{l}, \theta_{\bar{l}}\right\}$ in Fig. 8, for different values of the dimensionless 
stiffness $q_{1}$, taken equal to $0.7,1.1$, and 2 . Note that solid (dashed) lines represent stable (unstable) configurations.
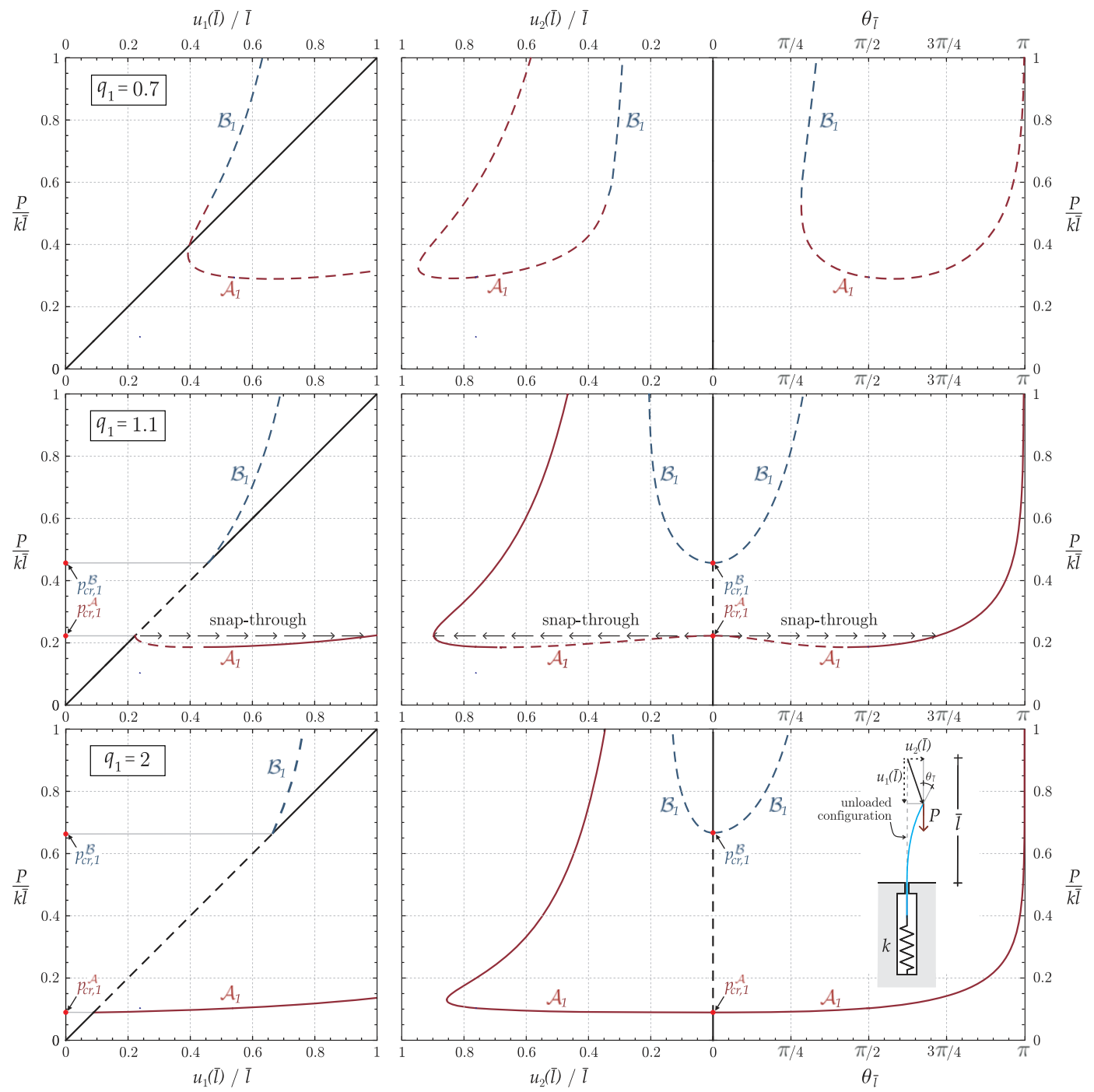

Figure 8: First-mode of bifurcation: deformation paths of the structure sketched in the inset, expressed as the (dimensionless) applied load versus (dimensionless) displacement components and rotation of the blade loaded edge.

The following observations can be drawn.

- The upper part of Fig. 8 is relative to $q_{1}=0.7$. In this case, no bifurcation occurs and the blade rigidly penetrates into the sliding sleeve. Note that alternative (and unstable) equilibrium configurations exist in addition to the trivial straight configuration, but (with the exception of the figure on the left, which can give a false impression of bifurcation) they do not cross (and even 'touch') the trivial path, so that these non-trivial unstable configurations cannot spontaneously be reached by the system. 
- Both the central and the lower parts of Fig. 8 show two bifurcation loads associated to the first bifurcation mode and show the restabilization of the straight configuration after the second buckling load.

- The central part of Fig. 8, referred to $q_{1}=1.1$, shows an example of a descending path of the lower equilibrium branch, associated to a snap-through of the system. On the other hand, the lower part of Fig. 8, referred to $q_{1}=2$, shows that the lower equilibrium branch has always a positive slope (so that snap-through does not occur).

- Comparing the central and the lower parts of Fig. 8 we may observe that an increase in the stiffness of the system decreases the buckling load and increases the restabilization load.

- The fact that a vertical Eshelby-like force is generated and 'expels' the blade from the sliding sleeve after buckling is not directly visible in Fig. 8. The visualization of this effect requires plotting $P$ as a function of $l_{e q}$, which is done in Fig. 10 where comparison with experiments is presented.

An example of bifurcation paths involving the first, the second and the third mode is reported in Fig. 9, where the load $P$ (made dimensionless through division by $k \bar{l}$ ) is plotted as a function of the rotation of the loaded end of the blade, $\theta_{\bar{l}}$. In the three parts of Fig. 9 , referring (from left
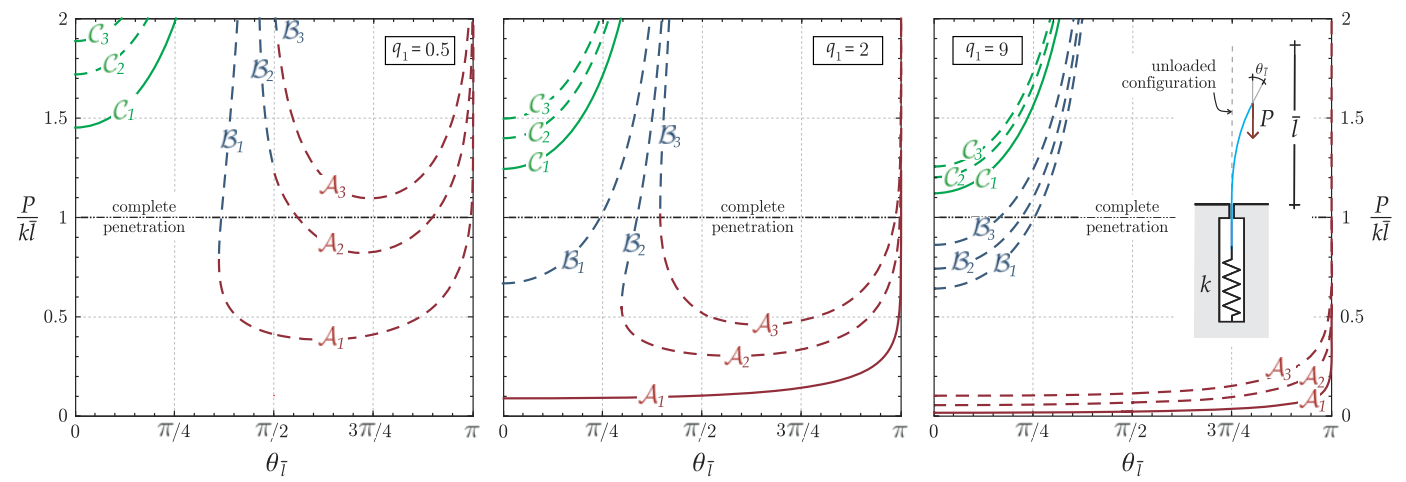

Figure 9: Force vs. blade's edge rotation of the structure sketched in the inset, evidencing the first, second and third mode of bifurcation. The threshold marked as 'complete penetration' corresponds to the complete penetration of the blade into the sliding sleeve. In the example on the left there is no bifurcation, while in the central part of the figure only the first mode is involved. In the right part of the figure, the first, second and third mode of bifurcation may occur before the 'complete penetration' is attained.

to right) to $q_{1}$ equal to $0.5,2$, and 9 , a line denoted as 'complete penetration' is reported at the value of parameter $p=P /(k \bar{l})=1$, corresponding to the complete penetration of the blade into the sliding sleeve. Therefore, curves reported beyond that limit are mathematically corrected, but meaningless from mechanical point of view. A situation where the postcritical paths do not intersect the vertical axis (corresponding to stable equilibrium only for the trivial straight configuration), and thus bifurcation does not occur, is reported in the left part of the figure, $q_{1}=0.5$. Here, firstand second-mode non-trivial unstable configurations exist below the 'complete penetration' limit but these cannot be reached during loading of the system. Bifurcation is possible in the central, 
$q_{1}=2$, and right, $q_{1}=9$, parts of the figure. While only first mode bifurcations occur in the former case, in the latter case first, second and third modes bifurcations are possible. In general, all modes superior to the first have been always found unstable in all cases analyzed (not only in those reported in this article).

An essential part in the present study is to show that all the found mechanical behaviours can be realized in practice. To this purpose, we have designed, realized and tested model structures to verify the theoretical findings. We have already anticipated with Figs. 3-6 that the theory has been fully confirmed, so that our intention is now to provide quantitative support.

Two prototypes (called 'Prototype 0', see Appendix A and Fig. 11, and 'Prototype 1', Fig. 2, right) of the structure sketched in Fig. 1 have been realized, according to the design scheme shown in Fig. 2 (left). Both prototypes have fully confirmed the theory, although Prototype 0 only in a qualitative way, so that Prototype 1 has been later manufactured to obtain quantitative results.

The linear elastic spring represented in Fig. 1 has been realized by hanging a highly-stiff horizontal bar (to which the elastic blade is clamped) with two metal springs. The horizontal bar can only rigidly translate as constrained by two linear bushings (LHFRD12, Misumi Europe).

Five blades have been employed (lengths: $600 \mathrm{~mm}, 530 \mathrm{~mm}, 510 \mathrm{~mm}, 430 \mathrm{~mm}$, and $360 \mathrm{~mm}$ ) with the 'stiff' springs and four blades (lengths: $600 \mathrm{~mm}, 530 \mathrm{~mm}, 510 \mathrm{~mm}$ and $430 \mathrm{~mm}$ ) with the 'compliant' one, all realized with C62 carbon-steel strips ( $25 \mathrm{~mm} \times 2 \mathrm{~mm}$ cross section) in the experiments. For these blades the bending stiffness $B$ has been determined with flexure experiments to be equal to $2.70 \mathrm{Nm}^{2}$. The same sliding sleeve employed by Bigoni et al. (2013) has been used, which is $296 \mathrm{~mm}$ in length and has been realized with 27 pairs of rollers (made up of $10 \mathrm{~mm}$ diameter and $15 \mathrm{~mm}$ length teflon cylinders, each containing two roller bearings). The tolerance between the metal strip and the rollers has been set to be $0.2 \mathrm{~mm}$. Two pairs of carbon steel (EN 10270-1 SH) springs (the so-called 'stiff': D19130, $1.6 \mathrm{~mm}$ wire diameter and $12.5 \mathrm{~mm}$ mean coil diameter, $k=600 \mathrm{~N} / \mathrm{m}$; and the so-called 'compliant': D19100 $1.25 \mathrm{~mm}$ wire diameter and $8 \mathrm{~mm}$ mean coil diameter, $k=540 \mathrm{~N} / \mathrm{m}$, purchased from D.I.M.) have been used. The dead load at the end of the blade has been imposed by filling (at a constant rate of $10 \mathrm{~g} / \mathrm{s}$ ) two containers with water and has been measured with two miniaturized Leane XFTC301 (R.C. 500N) load cells. The penetration length $l_{e q}$ has been obtained by measuring the displacement of the lower edge of the blade through a magnetic noncontact displacement transducer GC-MK5 (from Gemac).

Experimental results (solid lines) are reported in Fig. 10 and compared with theoretical predictions (dashed lines). Results are expressed in terms of applied load $P$ (measured in N) as a function of the amount of blade internal to the sliding sleeve, $l_{e q}$ (measured in $\mathrm{cm}$ ). Results reported on the left refer to 'compliant' spring, $k=540 \mathrm{~N} / \mathrm{m}$, while results reported on the right to the 'stiff' one, $k=600 \mathrm{~N} / \mathrm{m}$. Four blades of different length have been used in the former case, five in the latter. For both spring stiffnesses, the blades with shortest length show a pure translation without buckling, while buckling has been observed for all the other lengths. A snap-through behaviour was theoretically predicted for $\bar{l}=51 \mathrm{~cm}$ of the 'compliant' spring case and for $\bar{l}=43 \mathrm{~cm}$ of the 'stiff' spring case. In the former case $(\bar{l}=51 \mathrm{~cm})$, the descending postcritical path is so short and weakly inclined that it becomes hardly visible in the graph and therefore the snap-through has not been experimentally observed. On the other hand, in the latter case $(\bar{l}=43 \mathrm{~cm})$ the snap-through has been so violent that the experiment has been immediately interrupted and subsequent data have not been measured.

A very nice agreement between theory and experiments (fully confirming the presence of the 

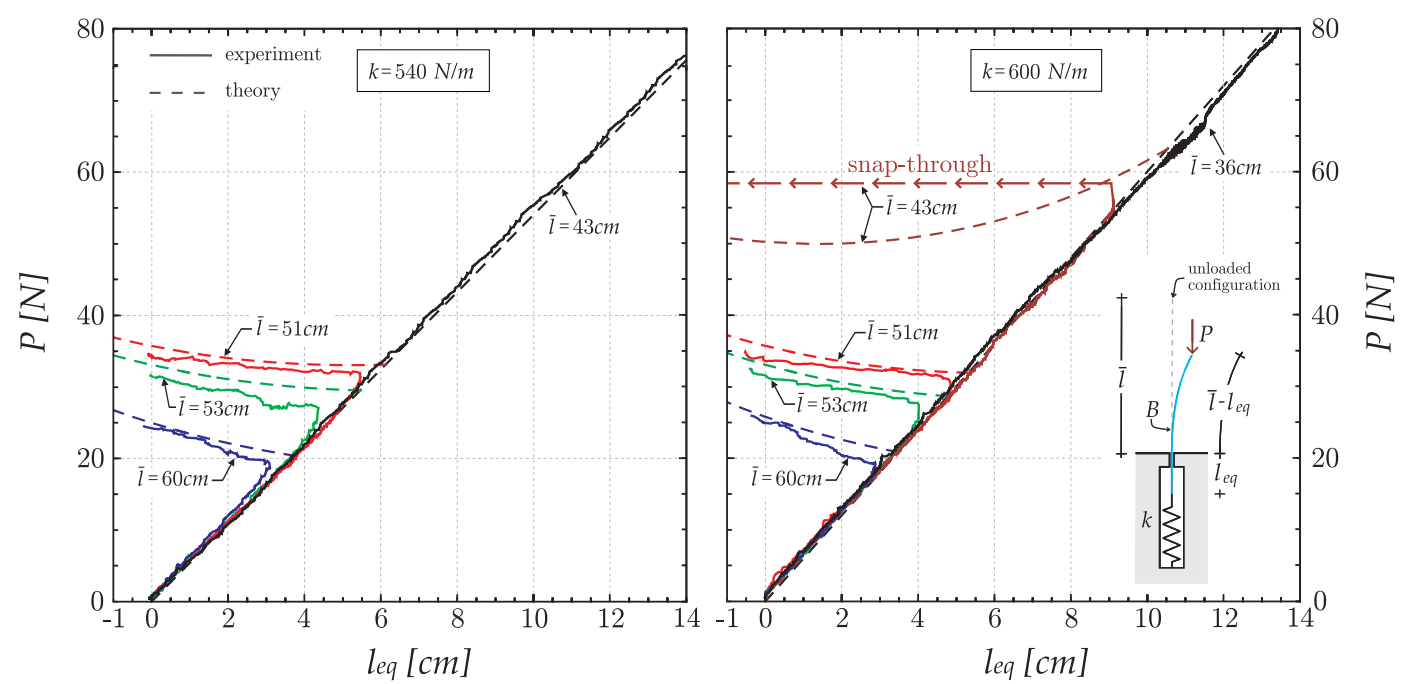

Figure 10: Comparison of theoretical and experimental results obtained with the set-up shown in Fig. 2.

Eshelby-like force) can be noted from Fig. 10, with departures from the straight configuration observed to occur slightly before the theoretical loads for buckling, which is nothing but the usual effect of the imperfections (see for instance Bigoni et al. 2012). Experiments clearly show that the blade is ejected from the sliding sleeve (which corresponds to a decrease in $l_{e q}$ ) during the postcritical behaviour of the structure and that the measured values of the force perfectly agree with those found by Bigoni et al. (2013).

Finally, the theoretical and experimental shapes of the elastica are so tightly close to each other that result superimposed in the the photos shown in Figs. 4, 5 and 6. Therefore, the calculated elastica curves have been omitted from the figures to preserve the view of the experiment.

Movies of the experiments can be found in the additional material at http://ssmg.unitn.it/.

\section{Conclusions}

A number of unexpected features can be observed in the bifurcation and instability behaviour of elastic systems containing elements capable of developing Eshelby-like forces, as for instance a sliding sleeve. The Eshelby-like force explains surprising equilibrium configurations (as that shown in Fig. 6) and concurs in determining restabilization of the straight configuration (Fig. 4). These features have been theoretically and experimentally demonstrated and are now awaiting for exploitation in the design of flexible systems and extremely deformable structures. In particular, applications can be envisaged to deformable systems showing restabilization, thus allowing the possibility of realizing cyclic motions with elastic elements. 


\section{APPENDIX A. Prototype 0 and further details on the experimental setup}

The Prototype 0 (Fig. 11) has been realized to provide a qualitative experimental validation of the features displayed by the mechanical system shown in Fig. 1.
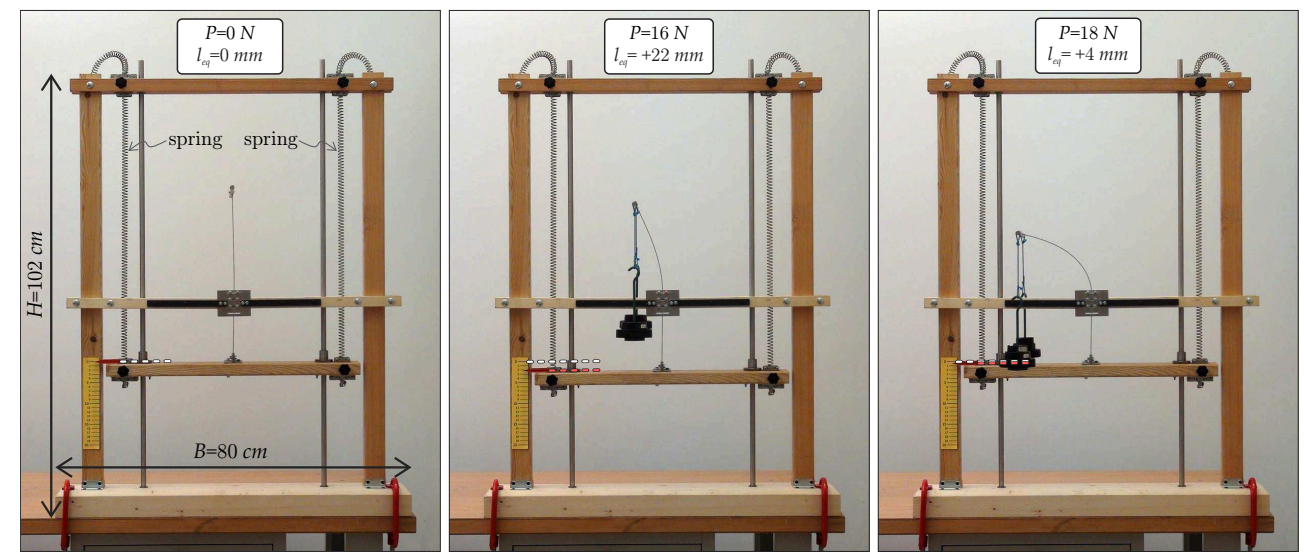

Figure 11: The unloaded configuration (left) and two deformed configurations (center and right) of Prototype 0. At high load (right) the base of the structure has an upward rigid translation when compared with the configuration at low load (left), a clear indication of the presence of the Eshelby-like force.

Two blades have been employed (with lengths $250 \mathrm{~mm}$ and $200 \mathrm{~mm}$ ), both realized with a C62 carbon-steel strip $(25 \mathrm{~mm} \times 1 \mathrm{~mm}$ cross section). The sliding sleeve, $31 \mathrm{~mm}$ in length, has been realized with three pairs of rollers $(15 \mathrm{~mm}$ diameter teflon cylinders, each containing two roller bearings). Three pairs of carbon steel (EN 10270-1 SH) springs (D19060, $0.8 \mathrm{~mm}$ wire diameter and $5 \mathrm{~mm}$ mean coil diameter; D19130, $1.6 \mathrm{~mm}$ wire diameter and $12.5 \mathrm{~mm}$ mean coil diameter; D19100, $1.25 \mathrm{~mm}$ wire diameter and $8 \mathrm{~mm}$ mean coil diameter all purchased from D.I.M.) have been used. The two linear bushings (LHFRD12) used to maintain horizontal the bar to which the blade is clamped have been purchased from Misumi Europe. Load has been controlled by manually imposing given weights.

For both Prototypes 0 and 1, all the experimental tests have been performed in a controlled temperature $\left(20 \pm 0.2^{\circ} \mathrm{C}\right)$ and humidity $(48 \pm 0.5 \%)$ room and data have been acquired with a NI CompactDAQ system, interfaced with Labview 8.5.1 (National Instruments). Furthermore, photos have been taken with a Sony NEX 5N digital camera, equipped with 3.5-5.6/18-55 lens (optical steady shot from Sony Corporation) and movies have been recorded during the tests with a Sony handycam (model HDR-XR550VE).

Additional material can be found at http://ssmg.unitn.it/.

Acknowledgments Financial support from the European FP7 - Intercer2 project (PIAP-GA-2011286110-INTERCER2) is gratefully acknowledged. 


\section{References}

[1] Balabukh, L.I., Vulfson, M.N., Mukoseev, B.V. and Panovko, Ya. G. (1970). On work done by reaction forces of moving supports. In: Research on Theory of Constructions 18, 190-200, Moscow.

[2] Bertoldi, K. and Boyce, M.C. (2008) Mechanically triggered transformations of phononic band gaps in periodic elastomeric structures. Phys. Rev. B 77, 052105.

[3] Bigoni, D. (2012) Nonlinear Solid Mechanics. Bifurcation theory and material instability, Cambridge University Press.

[4] Bigoni, D., Misseroni, D., Noselli, G. and Zaccaria, D. (2012) Effects of the constraints curvature on structural instability: tensile buckling and multiple bifurcations. Proc. R. Soc. A 468, 21912209 .

[5] Bigoni, D. Dal Corso, F. Bosi, F. Misseroni, D. (2013) Eshelby-like forces acting on elastic structures: theoretical and experimental proof. Mech. Materials, doi: 10.1016/j.mechmat.2013.10.009

[6] Bigoni, D and Noselli, G. (2011) Experimental evidence of flutter and divergence instabilities induced by dry friction. J. Mech. Phys. Solids 59, 2208-2226.

[7] Broman, A. (1970). Introduction to Partial Differential Equations: From Fourier Series to Boundary-Value Problems, Dover.

[8] Chan, E.P., Smith, E., Hayward, R., Crosby, A.J. (2008) Surface Wrinkles for Smart Adhesion. Advanced Materials, 20, 711-716.

[9] Courant, R. and Hilbert, D. (1953) Methods of Mathematical Physics, Wiley.

[10] Eshelby JD (1956) The continuum theory of lattice defects. In Progress in Solid State Physics 3 (eds. F. Seitz and D. Turnbull) 79-144, Academic Press, New York.

[11] Feodosyev, V.I. Selected Problems and Questions in Strength of Materials. MIR, Moscow, 1977.

[12] Li, J., Shim, J., Deng, J., Overvelde, J.T.B., Zhu, X., Bertoldi, K. and Yang, S. (2012) Switching periodic membranes via pattern transformation and shape memory effect. Soft Matter 8, 10322-10328.

[13] Majidi, C., 2007. Remarks on formulating an adhesion problem using Eulers elastica. Mech. Res. Comm. 34, 8590.

[14] Majidi, C., O'Reilly, O.M., Williams, J.A. (2012) On the stability of a rod adhering to a rigid surface: Shear-induced stable adhesion and the instability of peeling. J. Mech. Phys. Solids 60, $827-843$.

[15] Potier Ferry, M. (1987) Foundations of elastic postbuckling theory. In Buckling and postbuckling: four lectures in experimental, numerical, and theoretical solid mechanics. Eds. J. Arbocz, M. Potier-Ferry, J. Singer, V. Tvergaard, Lecture notes in physics, Springer-Verlag, 288, $1-82$.

[16] Rogers, J.A., Someya, T. and Huang, Y. (2010) Materials and mechanics for stretchable electronics. Science 327, 1603-1607. 
[17] Shim, J., Perdigou, C., Chen, E.R., Bertoldi, K., Reis, P.M. (2012) Buckling-induced encapsulation of structured elastic shells under pressure. Proc. Natl. Acad. Sci. U.S.A. 109, 5978-5983.

[18] Tarnai, J. (1980). Destabilizing effect of additional restraint on elastic bar structures. Int. J. Mech. Sci. 22, 379-390.

[19] van Brunt, B. (2005) The calculus of variations. Springer. 